Proceedings

\title{
A Sensor Platform for Smart Hydrogels in Biomedical Applications ${ }^{\dagger}$
}

\author{
Julia Körner 1, $\ddagger$, , Christopher Friedrich Reiche ${ }^{1, \ddagger}$, Hsuan-Yu Leu ${ }^{2}$, Navid Farhoudi ${ }^{1}$, \\ Jules Magda ${ }^{2}$ and Florian Solzbacher ${ }^{1}$ \\ 1 Department of Electrical and Computer Engineering, University of Utah, Salt Lake City, UT 84112, USA; \\ christopher.reiche@utah.edu (C.F.R.); navid.farhoudi@utah.edu (N.F.); florian.solzbacher@utah.edu (F.S.) \\ 2 Department of Chemical Engineering, University of Utah, Salt Lake City, UT 84112, USA; u1011614@utah.edu \\ (H.-Y.L.); jj.magda@utah.edu (J.M.) \\ * Correspondence: julia.koerner2k@gmail.com \\ + Presented at the Eurosensors 2018 Conference, Graz, Austria, 9-12 September 2018. \\ $\ddagger$ These authors contributed equally to this work.
}

Published: 3 December 2018

\begin{abstract}
Smart hydrogels are inherently biocompatible hydrophilic three-dimensional polymer networks able to undergo a volume-phase transition in the presence of an analyte. By molecular imprinting and/or aptamer-based approaches they can be tailored for a wide range of analytes with high selectivity. In combination with the biocompatibility, this makes hydrogels very promising candidates for biomedical sensor applications. However, to date hydrogels are rarely used for that purpose as the reliable detection of their swelling state remains a challenge. Here we report on a newly developed biocompatible bending sensor platform which can be equipped with almost any smart hydrogel, thereby paving the way for biomedical applications.
\end{abstract}

Keywords: smart hydrogel; biomedical sensor; mechanical bending

\section{Introduction}

The creation of a biomedical sensor device requires the ability to reliably detect very small quantities of a target analyte as well as biocompatibility, long-term stability, robustness in an in-vivo environment and the possibility of sterilization. While this is by far not a complete list of requirements, it already illustrates the challenge for biomedical sensor development.

A very promising approach for specific analyte detection are certain polymers, so called smart hydrogels. These are hydrophilic three-dimensional polymer networks and due to their high water content $(\geq 90 \%)$ and mechanical properties similar to tissue, they are inherently biocompatible [1]. A smart hydrogel reacts to an external parameter, e.g., temperature, $\mathrm{pH}$, ion (charge) concentration change, by a volume-phase-transition, resulting in a change in the volume and stiffness of the polymer [2]. Additionally, the smart hydrogel's fabrication process can include molecular imprinting techniques and/or the incorporation of additional functional groups into the polymer network, in order to bind a desired analyte with very high selectivity $[3,4]$. Thus, their biocompatibility and versatility make them very interesting candidates for biosensing.

However, the challenge lies in the reliable detection of the hydrogel's swelling state. Any sensor device should be as biocompatible as the hydrogel itself and it should be sensitive to smallest changes of the hydrogel's swelling state. Ideally, the detection concept is not limited to one specific analyte or hydrogel but merely constitutes a sensor platform that can then be employed for any type of smart hydrogel. Various hydrogel-based sensing approaches have been reported, including the use of fluorescence [5], magnetic nanoparticles [6], silicon pressure sensors [7] and cantilevered 
structures which bend due to the hydrogel's volume change [8]. The main challenges of these approaches are often a limitation to a specific analyte (e.g., in case of fluorescence) which restricts the versatility, or issues with biocompatibility (e.g., in case of silicon pressure sensors or magnetic particles).

Here we report on a recent development of a biomedical sensing platform which addresses the aforementioned challenges and is compatible with different hydrogels for a variety of analytes and not limited to one specific biomarker or molecule. We describe the design and first measurements of such a platform which is based on a mechanical thin film polymer bending sensor with electrical readout.

\section{Bending Sensor Concept}

The sensor platform comprises a sensing structure and a smart hydrogel. The sensing structure is created by a polyimide thin film (few $\mu \mathrm{m}$ thickness), which acts as substrate for fabrication, and biocompatible encapsulation material, and that contains an embedded metallic lead pattern $(200 \mathrm{~nm}$ thickness). In order to add analyte sensitivity, a smart hydrogel is deposited in micro strips on one side of the polymer substrate by an adhesion promoting process [9]. Hydrogel expansion or contraction bends the sensing structure, thereby altering the electrical impedance of the embedded metallic leads on deformation (Figure 1). Given a suitable calibration procedure, this change in impedance can be related to the analyte concentration. Currently, the impedance change is measured cable-based with a network analyzer. However, this is only an intermediate step as the sensor design creates the opportunity of incorporating it into a wireless readout scheme.
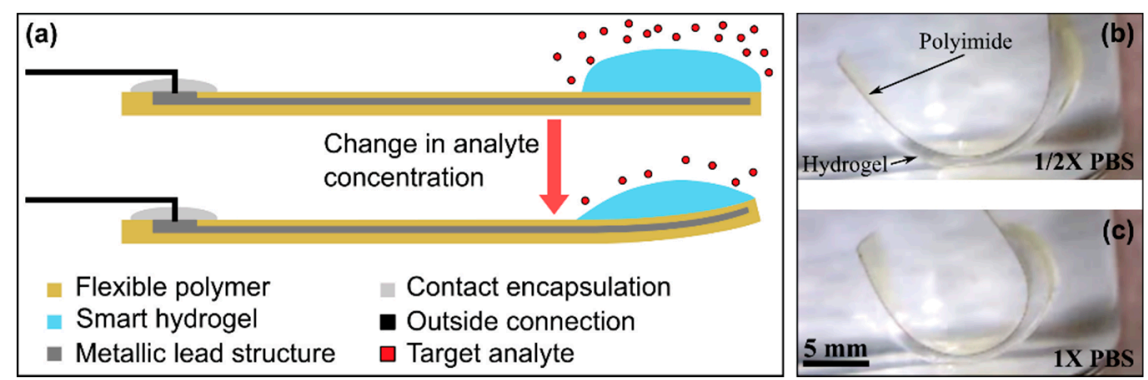

Figure 1. (a) Basic bending sensor concept, $(\mathbf{b}, \mathbf{c})$ polyimide sensing structure with hydrogel (no electrical contacts) immersed in different salt concentrations of phosphate buffered saline (PBS) solution to demonstrate the visual change in bending. The hydrogel is attached to the bottom side of the polyimide as indicated.

The sensing principle is independent of the specific type of smart hydrogel used because it is only based on the mechanical deformation induced in the polymer-metal sandwich. This universality in terms of the target analyte in combination with the biocompatible materials and the potential for miniaturization and wireless readout are a considerable advantage of the concept compared to other approaches $[2,5,6,10]$ for a wearable or implantable biomedical hydrogel sensor.

Furthermore, our concept features geometric flexibility (a wide variety of form factors can easily be fabricated) and low manufacturing costs. In comparison to the previously published pressure sensor approach [10], the hydrogels employed on the polyimide sensing part are much thinner (tens vs. hundreds of $\mu \mathrm{m}$ ), accelerating diffusion and, consequently, reducing sensor response time (seconds vs. minutes/hours), making the device fast enough for real-time monitoring of biomarker and drug levels in blood.

\section{Results and Discussion}

\subsection{Experimental Results}

In order to evaluate the sensor concept, test devices were fabricated (Figure 2a) with a $200 \mu \mathrm{m}$ thick acrylamide-based smart hydrogel [9] and tested for ionic strength response. The fabrication 
process involves standard microfabrication steps such as deep-reactive ion etching (DRIE) and sputter deposition of metal.

For evaluation of sensor response, the change of the reflected amplitude (S11 parameter) at one of the sensor's initial resonance frequencies has been measured over time (Figure $2 b$ ) by alternating the solution between de-ionized water (DIW) and 1X phosphate-buffered saline (PBS) solution. In the post processing steps, the data was corrected for the offset induced by exchanging the liquid. This was done by averaging the last ten data points for each solution (when the sensor reached equilibrium) and then calculating the difference between this average value and the first data point of the next step. This gives an offset which is subtracted from all following data points.

We observed a clear sensor response almost immediately and within five minutes equilibrium is reached, thus demonstrating the functionality of the sensor concept.
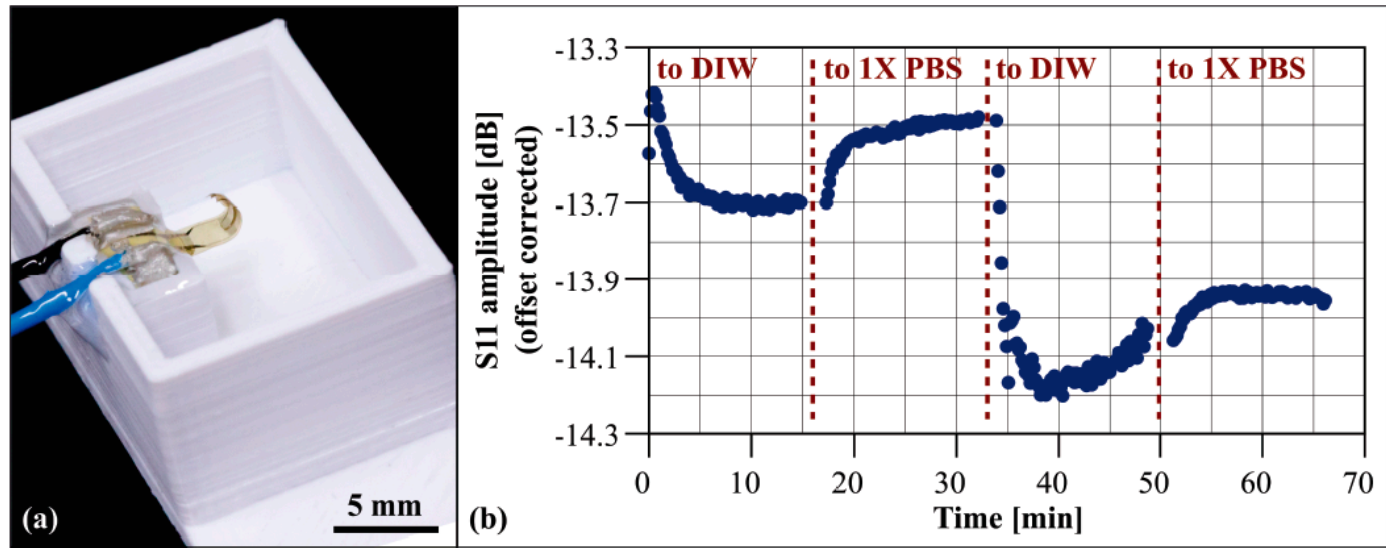

Figure 2. (a) Test set up for polyimide sensors (not immersed in solution for better visibility), (b) electrical sensor response for varying ionic strength in surrounding solution (alternated between deionized water (DIW) and phosphate-buffered saline (1X PBS)) (from data previously published in [11]). The measured signal is the reflective coefficient (S11 parameter) obtained with a network analyzer at a frequency of $163.5 \mathrm{MHz}$. A slight offset induced through the liquid exchange has been subtracted.

\subsection{Sensor Design}

Challenges which need to be addressed during further sensor development include optimization of the sensing structure for different smart hydrogels. Depending on the target analyte and the composition, the smart hydrogel's volume change differs, and, consequently, the force it can exert onto the bending structure varies. Therefore, it is crucial to adapt the bending stiffness of the sensing structure for these different hydrogels. In order to gain insight in the basic working principles of the sensing structure, finite element simulations are used to optimize the bending geometry for specific smart hydrogel composition and to study the dependencies and variables, e.g., shape of the sensing structure, polymer substrate thickness in relation to the hydrogel thickness and dimensions and geometry of the metal layer structure.

\section{Conclusions and Outlook}

We developed a new biocompatible sensor platform based on smart hydrogels and a flexible polymer film for biomedical applications. We fabricated the first sensor devices and measured their response to changes in the ionic concentration of a liquid. These first proof-of-principle experiments demonstrate the viability and potential of our approach and are the basis for further development.

Future experimental steps include sensor testing with smart hydrogels sensitive to different analytes (e.g., glucose, thrombin) and optimization of the incorporated lead structure as that mainly defines the sensor's signal in response to bending. We are furthermore working on devices with varying form factors for a catheter application. In this concept, the polyimide structure is integrated into a standard catheter and due to its small dimensions it only fills $2-3 \%$ of the inner lumen of the 
sheath. Therefore, the catheter can be used as before, i.e., neither significant changes in clinical procedures nor additional punctures for the patient are necessary. The benefit of this sensor is that it will allow monitoring of bioanalyte levels directly in blood and almost in real-time which gives the clinician more information about the current status of a patient and could help prevent deaths, e.g., due to inadequate dosage of anesthesia.

Author Contributions: Conceptualization, J.K., C.F.R., J.M. and F.S.; Funding acquisition, F.S., J.K., C.F.R. and J.M.; Supervision, F.S., J.M., J.K. and C.F.R.; Project administration, J.K. and C.F.R.; Investigation, J.K., C.F.R., N.F. and H.-Y.L., Resources, F.S. and J.M.; Writing - original draft, J.K.; Writing - review and editing, J.K. and C.F.R.

Funding: This research was funded by the Joe W. and Dorothy Dorsett Brown Foundation, the Olive Tupper Foundation, Sentiomed, Inc., the CARMA Center of the University of Utah and the University of Utah School of Dentistry.

Acknowledgments: The authors would like to thank Wonmi Lee for supporting work regarding hydrogel preparation.

Conflicts of Interest: Florian Solzbacher declares financial interest in Blackrock Microsystems LLC and Sentiomed, Inc. Jules Magda declares financial interest in Applied BiosensorsLLC.

\section{References}

1. Peppas, N.A.; Hilt, J.Z.; Khademhosseini, A.; Langer, R. Hydrogels in Biology and Medicine: From Molecular Principles to Bionanotechnology. Adv. Mater. 2006, 18, 1345-1360, doi:10.1002/adma.200501612.

2. Richter, A.; Paschew, G.; Klatt, S.; Lienig, J.; Arndt, K.F.; Adler, H.J.P. Review on Hydrogel-based pH Sensors and Microsensors. Sensors 2008, 8, 561-581, doi:10.3390/s8010561.

3. Zhang, Z.; Liu, J. Molecularly Imprinted Polymers with DNA Aptamer Fragments as Macromonomers. Appl. Mater. Interfaces 2016, 8, 6371-6378, doi:10.1021/acsami.6b00461.

4. Lin, G.; Chang, S.; Hao, H.; Tathireddy, P.; Orthner, M.; Magda, J.; Solzbacher, F. Osmotic Swelling Pressure Response of Smart Hydrogels Suitable for Chronically-Implantable Glucose Sensors. Sens. Actuators B Chem. 2010, 144, 332-336, doi:10.1016/j.snb.2009.07.054.

5. Cappon, G.; Acciaroli, G.; Vettoretti, M.; Facchinetti, A.; Sparacino, G. Wearable Continuous Glucose Monitoring Sensors: A Revolution in Diabetes Treatment. Electronics 2017, 6, 65, doi:10.3390/electronics6030065.

6. Nguyen, T.; Cho, S.; Bhola, V.; Ko, S.; Sharma, R.; Magda, J.; Tathireddy, P. Sensor-Array for Continuous Monitoring of Biochemicals for Bioprocess Control. In Proceedings of the 18th International Conference on Solid-State Sensors, Actuators, and Microsystems, Transducers, Anchorage, AK, USA, 21-25 June 2015; p. 1684, doi:10.1109/TRANSDUCERS.2015.7181267.

7. Orthner, M.P.; Buetefisch, S.; Magda, J.; Rieth, L.W.; Solzbacher, F. Development, Fabrication, and Characterization of Hydrogel Based Piezoresistive Pressure Sensors with Perforated Diaphragms. Sens. Actuators A Phys. 2010, 161, 29-38, doi:10.1016/j.sna.2010.05.023.

8. Zhang, Y.; Ji, H.F.; Snow, D.; Sterling, R.; Brown, G. A pH Sensor Based on a Microcantilever Coated with Intelligent Hydrogel. Instrum. Sci. Technol. 2004, 32, 361-369, doi:10.1081/CI-120037668.

9. Horkay, F.; Cho, S.H.; Tathireddy, P.; Rieth, L.; Solzbacher, F.; Magda, J. Thermodynamic Analysis of the Selectivity Enhancement Obtained by Using Smart Hydrogels That Are Zwitterionic When Detecting Glucose with Boronic Acid Moieties. Sens. Actuators B Chem. 2011, 160, 1363-1371, doi:10.1016/j.snb.2011.09.079.

10. Orthner, M.P.; Lin, G.; Avula, M.; Buetefisch, S.; Magda, J.; Rieth, L.W.; Solzbacher, F. Hydrogel based sensor arrays $(2 \times 2)$ with perforated piezoresistive diaphragms for metabolic monitoring (in vitro). Sens. Actuators B Chem. 2010, 145, 807-816, doi:10.1016/j.snb.2010.01.063.

11. Körner, J.; Reiche, C.F.; Leu, H.Y.; Magda, J.; Solzbacher, F. Fast-reacting smart hydrogel-based sensor platform for biomedical applications. TechConnect Briefs 2018, 3, 206-208. 